\title{
Hypersonic Flow of Rarefied Gas Near the Brazilian Satellite During its Reentry into Atmosphere
}

\author{
Felix Sharipov \\ Departamento de Física, Universidade Federal do Paraná \\ Caixa Postal 19044, 81531-990 Curitiba, Brazil
}

Received on 17 January, 2003

\begin{abstract}
To calculate aerothermodynamic characteristics of the Brazilian satellite SARA (Portuguese abbreviation: SAtélite de Reentrada Atmosférica) during its re-entry the Direct Simulation Monte Carlo method is applied. The numerical calculations were carried out in a wide range of the gas rarefaction beginning from the free molecular regime up to the hydrodynamic one for three values of the Mach number equal to 5, 10 and 20. The following numerical data are presented in the paper: drag force of the satellite, distributions of the heat flux, pressure coefficient and skin friction coefficient over the satellite surface, the density and temperature distributions in the front of the satellite and streamlines in the gas around the satellite. To study the influence of the satellite temperature upon these characteristics some calculations were carried out for different values of the satellite temperature.
\end{abstract}

\section{Introduction}

To realize a re-entry of satellite into the atmosphere two main problems related to the gas dynamics must be solved. The first of them is the calculation of satellite trajectory based on knowledge about the drag force. The second problem is the creation of adequate heat protection based on information about the heat flux on the satellite surface. The complex of these problems is usually called as the aerothermodynamics of satellite [1-11].

In the high atmosphere stratum the mean free path of gaseous molecules has the order of several meters. In such conditions the gas flow can be considered as free-molecular, i.e. the intermolecular collisions can be neglected in calculations of the aerothermodynamic characteristics. Near the Earth surface the molecular mean free path is so small that the air around a satellite can be considered as a continuous medium. However, there is a significant layer of the atmosphere where the mean free path has the order of the satellite size. To calculate the aerothermodynamic characteristics of satellite passing through such a layer the intermolecular collisions can be already taken into account but the continuous mechanics equations cannot be applied yet. In such situation the methods of rarefied gas dynamics must be used, which can be divide in two large groups. The first group of the methods is based on the kinetic Boltzmann equation [1216], while the second approach is based on the Direct Simulation Monte Carlo (DSMC) method [17]. In spite of the fact that the second method requires greater computational efforts it is more suitable for practical calculations because it allows us easily to generalize the program for polyatomic gases and for gaseous mixtures. This generalization is very important because in practice one deals with the air, which is a mixture of polyatomic gases. So, in the present work the DSMC method was used to calculate the aerothermodynamic characteristics of SARA (Portuguese abbreviation: SAtélite de Reentrada Atmosférica). This is a Brazilian project of small reusable ballistic re-entry satellite, which will be used to realize scientific experiments in the microgravity conditions and to deliver their results to the Earth. The shape of the satellite are given in Fig. 1. Its sizes are as follows: $R=503.5 \mathrm{~mm}$ and $L=1410 \mathrm{~mm}$.

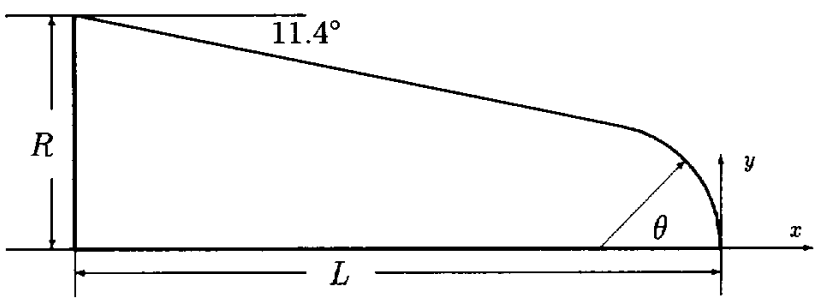

Figure 1. Shape of SARA.

The aim of the present work is to calculate the drag force, the heat flux on the satellite surface and other aerothermodynamic characteristics necessary to calculate the ballistic trajectory of the re-entry and to create an adequate thermal protection. The calculations are carried out over the whole range of the gas rarefaction embracing both free molecular and hydrodynamic regimes.

\section{Statement of the problem}

We consider an axisymmetric gas flow around the satellite assuming that the direction of the gas velocity $U_{\infty}$ far from the satellite coincides with its symmetry axis. We denote 
the temperature and the density of the undisturbed gas as $T_{\infty}$ and $\rho_{\infty}$, respectively. The solution of the problem is determined by the three main parameters:

(i) Mach number defined as

$$
\mathrm{Ma}=\frac{U_{\infty}}{c},
$$

where $c$ is the sound velocity of the gas given as

$$
c=\sqrt{\gamma \frac{k T_{\infty}}{m}},
$$

$k$ is the Boltzmann constant, $m$ is the gaseous molecular mass and $\gamma$ is the specific heat ratio.

(ii) Reynolds number defined as

$$
\operatorname{Re}=\frac{R U_{\infty} \rho_{\infty}}{\mu},
$$

where $R$ is the largest satellite radius (see Fig. 1), $\mu$ is the stress viscosity of the gas at the temperature $T_{\infty}$.

(iii) The ratio of the satellite temperature $T_{s}$ to the temperature of undisturbed gas $T_{\infty}$. Really, the satellite temperature $T_{s}$ must be calculated together with the flowfield of the gas. To realize such calculations we should know the thermodynamic characteristics of the satellite such as its thermal conductivity and specific heat. However, in the present work we are going to investigate the influence of the satellite temperature on the aerothermodynamic characteristics. For such a purpose we assume that the satellite temperature $T_{s}$ is given and it is uniform over the surface.

The gas rarefaction is usually characterized by the Knudsen number $(\mathrm{Kn})$ defined as

$$
\mathrm{Kn}=\frac{\lambda_{\infty}}{R},
$$

where $\lambda_{\infty}$ is the molecular mean free path corresponding to the temperature $T_{\infty}$ and density $\rho_{\infty}$. However, the Knudsen number can be related to the Mach number and Reynolds number via the expression of the viscosity. If we assume the hard sphere molecular model, then the viscosity obtained by the Chapman-Enskog method [15] reads

$$
\mu=\left(\frac{2 k T_{\infty}}{\pi m}\right)^{1 / 2} \rho_{\infty} \lambda_{\infty}
$$

Then, with the help of Eqs.(1) - (5) the relation of $\mathrm{Kn}$ to $\mathrm{Re}$ and $\mathrm{Ma}$ is obtained as

$$
\mathrm{Kn}=\left(\gamma \frac{\pi}{2}\right)^{1 / 2} \frac{\mathrm{Ma}}{\operatorname{Re}} .
$$

So, the Knudsen number is not independent parameter determining the solution of the problem but it just characterizes the gas rarefaction for given values of the Mach number and Reynolds number.

The thermodynamic characteristics to be calculated in the present paper are defined as follows:

Drag coefficient

$$
C_{D}=\frac{F}{P_{d} A}
$$

where $F$ is the drag force, $P_{d}$ is the dynamic pressure given as

$$
P_{d}=\frac{1}{2} \varrho_{\infty} U_{\infty}^{2}
$$

and $A=\pi R^{2}$ is the largest cross section of the satellite.

Coefficient of heat transfer

$$
C_{H}=\frac{q_{n}}{P_{d} U_{\infty}}
$$

where $q_{n}$ is the normal heat flux to the satellite surface.

Pressure coefficient

$$
C_{p}=\frac{P-P_{\infty}}{P_{d}}
$$

where $P$ is the normal pressure to the satellite surface and $P_{\infty}$ is the gas pressure far from the satellite given by the state equation

$$
P_{\infty}=\frac{k}{m} \rho_{\infty} T_{\infty}
$$

Skin friction coefficient

$$
C_{f}=\frac{\tau}{P_{d}}
$$

where $\tau$ is the tangential stress on the satellite surface.

Below, these characteristics will be given as a function of the three parameters: the Mach number (Ma), the Reynolds number $(\mathrm{Re})$ and the temperature ratio $T_{s} / T_{\infty}$.

\section{Method of solution}

The DSMC method consists of numerical simulation of movements and collisions of a large number of model particles [17]. The region of the gas flow is divided into a network of cells as is shown in Fig. 2. The dimension of the cells must be such that the change in flow properties across each cell would be small. Since we expect a significant variation of the properties near the satellite the cell size in this region is twice smaller than that far from the satellite.

The time is advanced in discrete steps of magnitude $\Delta t$, such that $\Delta t$ is small compared with the mean time between two successive collisions given by

$$
t_{m}=\lambda_{\infty}\left(\frac{m}{2 k T_{\infty}}\right)^{1 / 2}
$$

Initially, the model particles are distributed uniformly over the computational region with the Maxwellian distribution function. The positions and velocities of the particles must be saved in computer memory. Then, the particle motion and their collisions are uncoupled over the small time interval $\Delta t$ by repetition of the following two stages. 


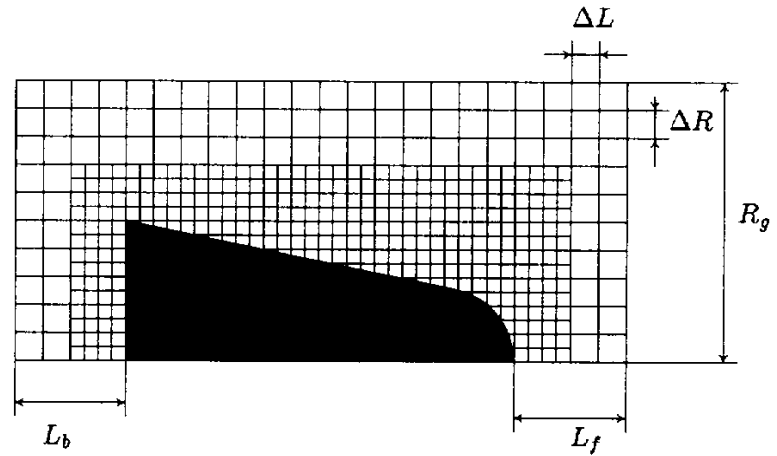

Figure 2. Numerical grid.

During the first stage the particles are moved through the distance determined by their velocities $\mathbf{v}$ and $\Delta t$. So the new coordinates of every particle are calculated via the old ones as

$$
\mathbf{r}_{\text {new }}=\mathbf{r}_{\text {old }}+\mathbf{v} \Delta t .
$$

If the trajectory crosses a solid surface a simulation of the gas-surface interaction is performed according to a given law. In the present work the diffuse reflection is assume, i.e. a particle is emitted by the surface in any direction with the same probability. Experimental data [18] showed that a deviation from the diffuse reflection is observed only for light gases, e.g. helium, and for an atomically clean surface, while the reflection on a contaminated surface is diffuse. In this stage the difference of momentum and kinetic energy of the particle before and after the collision with the surface is calculated. Then this information is used to calculate the drag coefficient $C_{D}$, the heat transfer coefficient $C_{H}$, the pressure coefficient $C_{p}$, and the skin friction coefficient $C_{f}$, at any surface point. New particles are generated at boundaries of the computational region with the distribution function corresponding to the bulk velocity $U_{\infty}$, density $\rho_{\infty}$ and temperature $T_{\infty}$ of the undisturbed gas. The particles going out from the computational region are removed from the computer memory.

On the second stage a number of pairs to be selected for intermolecular collisions is calculated as

$$
N_{\text {coll }}=N_{p} \bar{N}_{p} F_{N} \sigma c_{\max } \Delta t /\left(2 V_{C}\right),
$$

where $N_{p}$ is the number molecules in a cell during the last time interval $\Delta t, \bar{N}_{p}$ is the average value of $N_{p}$ during all previous intervals $\Delta t, F_{N}$ is the number of real gaseous particles represented by one model particle, $c_{\max }$ is a maximum relative velocity between two particles, $V_{C}$ is the cell volume, and $\sigma$ is the collision cross section of particles. Every selected pair is accepted for collision if $c / c_{\max }>R_{f}$, where $R_{f}$ is a random number varying from 0 to 1 and $c$ is the relative velocity for the given pair. Such a selection corresponds to the hard sphere model of molecules. If the pair is accepted then the pre-collision velocities of the particles are replaced by the post-collision values in accordance with the elastic collision law.

After a sufficient number of the repetitions of these two procedures we may calculate the macroscopic characteristics. The characteristics of our interest are calculated as: density

$$
\rho=\frac{m N_{p} F_{N}}{V_{C}}
$$

bulk velocity

$$
\mathbf{u}=\frac{1}{N_{p}} \sum_{i=1}^{N_{p}} \mathbf{v}_{i}
$$

temperature

$$
T=\frac{m}{3 k N_{p}} \sum_{i=1}^{N_{p}}\left(\mathbf{v}_{i}-\mathbf{u}\right)^{2} .
$$

As has been mentioned above the aerothermodynamic coefficients $C_{D}, C_{H}, C_{p}$ and $C_{f}$ are calculated during the simulation of the gas-surface interaction.

For the problem under consideration the numerical accuracy depends on many factors: size of the computational region determined by $R_{g}, L_{b}$ and $L_{f}$ (see Fig. 2), size of the cells determined by $\Delta L$ and $\Delta R$, number of the model particles $N$, time increment $\Delta t$. In the present work the following values of the grid parameters were used: $R_{g}=3 R$, $L_{f}=L, L_{b}=L, \Delta R=R / 12, \Delta L=L / 24$. The number of simulated particles was maintained about $10^{6}$. The time increment $\Delta t$ was 0.01 of the mean free time $t_{m}$

To verify the numerical accuracy some calculations were carried out for $\Delta t=0.002 t_{m}$ with the number of particles equal to $5 \times 10^{6}$. The parameters $R_{g}, L_{f}$ and $L_{b}$ were increased twice in other test calculations and the cell size, i.e. $\Delta L$ and $\Delta R$, were decreased twice. An analysis of the test calculations showed that the numerical accuracy of the drag coefficient $C_{D}$ was within $1 \%$, while the other aerothermodynamic characteristics were calculated with slightly larger numerical error, which does not exceed $5 \%$.

\section{Results and discussion}

The numerical calculations were carried out for several values of the Reynolds number $(\mathrm{Re})$ in the range from 0.1 to 4000. Three values of the Mach number (Ma) were considered: 5, 10 and 20. These are typical values of the Mach number during a re-entry of satellite. According to Eq. (6) the Knudsen number $(\mathrm{Kn})$ varied in the range from 200 to 0.001 , i.e. the free molecular, transition and hydrodynamic regimes were covered in the present work that corresponds to the conditions of satellite re-entry.

It is difficult to predict the temperature of the satellite surface $T_{s}$ during its re-entry. So it is possible just to estimate the influence of this temperature upon the aerothermodynamic characteristics. For this purpose the calculations were carried out for two values of the temperature ratio $T_{s} / T_{\infty}$, namely 1 and 10 . The first value corresponds to the situation when the temperature of the satellite $T_{s}$ is equal to the temperature of the undisturbed gas $T_{\infty}$. In this situation the satellite will be called as "cold". The second value $T_{s} / T_{\infty}=10$ corresponds to the situation when the satellite is significantly hotter then the undisturbed gas. Such a satellite will be called as "heated". The real temperature of the satellite surface $T_{s}$ is surely higher than the temperature of 
undisturbed gas $T_{\infty}$ because of the satellite heating. But $T_{s}$ cannot be higher than $10 \times T_{\infty}$ because at such a high temperature the satellite begins to fuse. So, considering these two values of the temperature ratio, i.e. $T_{s} / T_{\infty}=1$ and 10 , we can estimate the maximum variation of the aerothermodynamic characteristics due to the temperature of the satellite surface.

The numerical data on the drag coefficient $C_{D}$ for the cold satellite $\left(T_{s} / T_{\infty}=1\right)$ are presented in Fig. 3. One can see that the dependence of $C_{D}$ on the Mach number is very small in the hydrodynamic regime $(\mathrm{Re} \rightarrow \infty)$. It is also weak in the free molecular regime $(\mathrm{Re} \rightarrow 0)$. At the same time the dependence of the drag coefficient $C_{D}$ on the Reynolds number is significant.

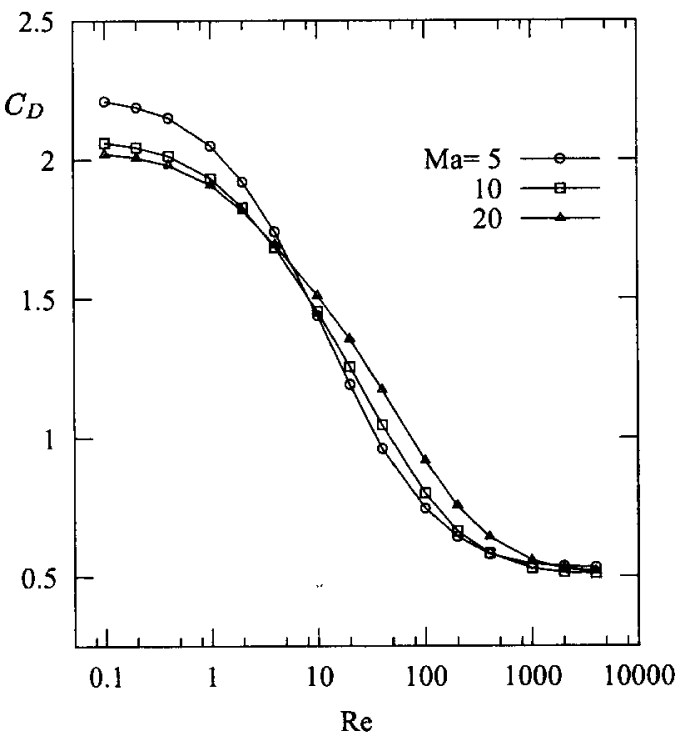

Figure 3. Drag coefficient $C_{D}$ vs Ma, Re at $T_{s} / T_{\infty}=1$

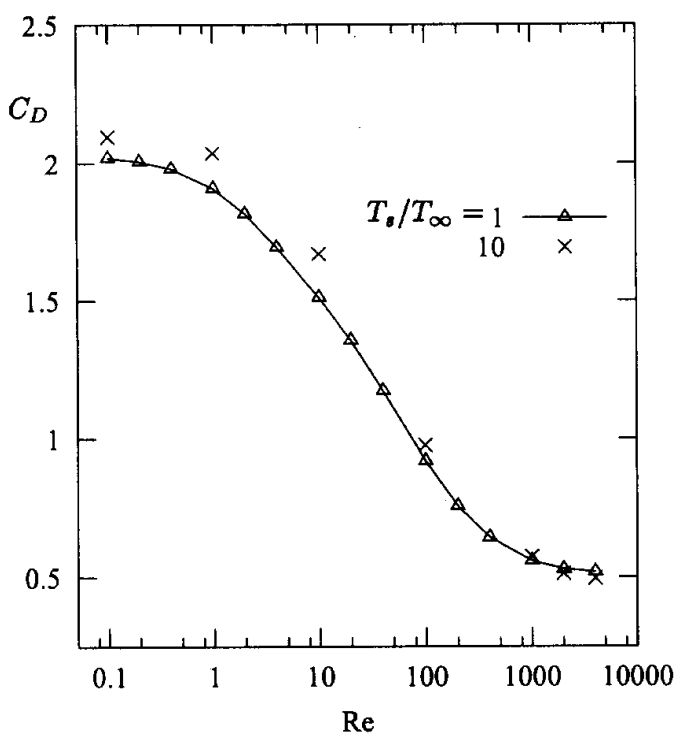

Figure 4. Drag coefficient $C_{D}$ vs $\mathrm{Re}$ and $T_{s} / T_{\infty}$ at $\mathrm{Ma}=20$

A comparison of the numerical values of the coefficient $C_{D}$ for the "cold" satellite $\left(T_{s} / T_{\infty}=1\right)$ and for the "heated" one $\left(T_{s} / T_{\infty}=10\right)$ is performed in Fig. 4 for the Mach number equal to 20. It can be seen that this influence is small. An analysis of the numerical data for $\mathrm{Ma}=5$ and 10 showed that in these cases the influence of $T_{s} / T_{\infty}$ upon the drag coefficient slightly larger but has the same order.

The coefficients $C_{H}, C_{p}$ and $C_{f}$ are given in Figs. 5, 6 and 7 , respectively, as a function the angle $\theta$ for the three values of the Mach number $\mathrm{Ma}=5,10$ and 20 for the two values of the temperature ratio $T_{s} / T_{\infty}=1$ and 10 and for the three values of the Reynolds number $\mathrm{Re}=0.1,10$ and 4000 . These values of Re represent the three regimes of flow: free molecular $(\mathrm{Re}=0.1)$, transition $(\mathrm{Re}=10)$ and hydrodynamic $(\operatorname{Re}=4000)$. The angle $\theta$ is defined in Fig. 1 .

The heat transfer coefficient $C_{H}$ given in Fig. 5. reaches its largest value at the front point of the satellite $\theta=0$. It depends significantly on the Reynolds number. Its dependence on the Mach number is weak if the satellite is "cold", i.e. $T_{s} / T_{\infty}=1$, while for the "heated" satellite, i.e. at $T_{s} / T_{\infty}=10$, the dependence on the Mach number can be significant. For example, at $\mathrm{Ma}=5$ and $\mathrm{Re}=4000$ the heat transfer coefficient $C_{H}$ is negative, while at $\mathrm{Ma}=10$ and $\mathrm{Ma}=20$ the coefficient is always positive. So, we may conclude that the influence of the satellite temperature $T_{s} / T_{\infty}$ on the heat transfer coefficient $C_{H}$ is significant at any Reynolds number if $\mathrm{Ma} \leq 5$, while for the high values of the Mach number, i.e. $\mathrm{Ma} \geq 10$ this influence is weak.

The numerical data on the pressure coefficient $C_{p}$ presented in Fig. 6 show that in the hydrodynamic regime $(\mathrm{Re}=4000)$ this coefficient is affected by neither satellite temperature $T_{s}$ nor Mach number. In the free molecular regime $(\mathrm{Re}=0.1)$ the coefficient $C_{p}$ significantly depends on $T_{s} / T_{\infty}$ for the low Mach number, i.e. $\mathrm{Ma}=5$, while for the high Mach number, i.e. $\mathrm{Ma}=20$, this dependence is weak. The coefficient $C_{p}$ reaches its maximum value in the front point $\theta=0$.

The dependence of the friction coefficient $C_{f}$ on the angle $\theta$ is shown in Fig. 7. One can see that this coefficient is weakly affected by the satellite temperature and by the Mach number, while it depends on the Reynolds number is strong, i.e. in the free molecular regime $(\mathrm{Re}=0.1)$ it is significantly larger than in the hydrodynamic regime $(\mathrm{Re}=4000)$. The coefficient $C_{f}$ reaches its maximum value at $\theta \approx 0.8 \mathrm{rad}$, i.e. $\theta \approx 45^{\circ}$, while in the front point $\theta=0$ it is naturally zero.

The density distribution along the symmetry axis $x$ is given in Fig. 8. The $x$-coordinate is shown in Fig. 1. These distributions show clearly the existence of the shock wave in front of the satellite in the hydrodynamic regime $(\mathrm{Re}=4000)$, i.e. for all values of the Mach number the drastic variation of the density occurs in the interval of $x / R$ from 0 to 0.2 . In the free molecular regime $(\mathrm{Re}=0.1)$ the density varies smoothly from its maximum value at $x / R=0$ to its equilibrium value $\rho_{\infty}$ by increasing $x / R$. In the transition regime $(\mathrm{Re}=10)$ the behavior of the density is intermediate. The density is affected significantly by the satellite temperature $T_{s}$ in the free molecular regime, while in the hydrodynamic regime the density distribution weakly depends on the satellite temperature. The density reaches its maximum value at the point $x=0$, which increases by increasing the Mach number. 

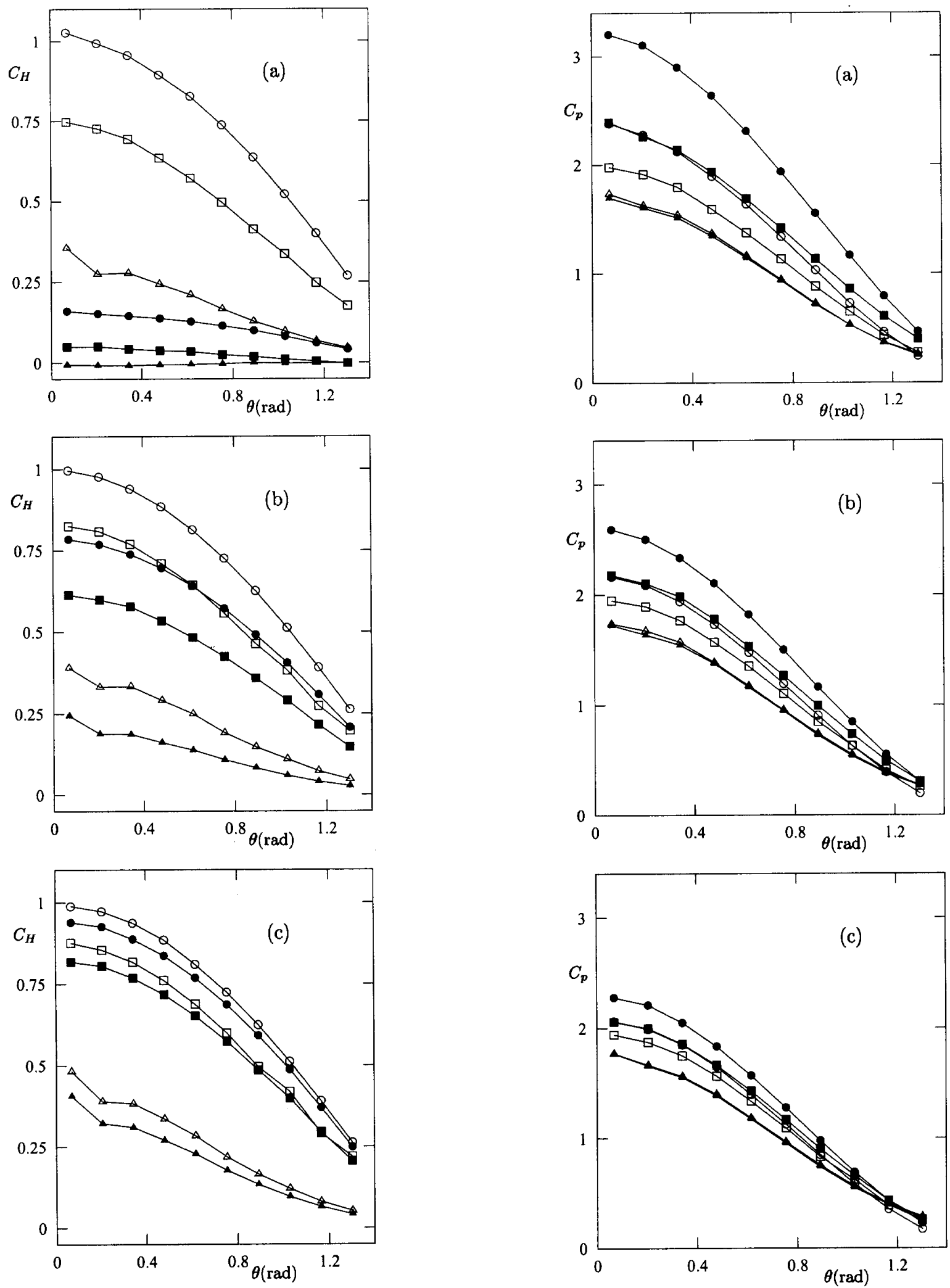

Figure 5. Heat transfer coefficient $C_{H}$ vs $\theta$ (rad): (a) - Ma=5, (b) - $\mathrm{Ma}=10$, (c) - $\mathrm{Ma}=20$; circles $-\mathrm{Re}=0.1$, squares - $\mathrm{Re}=10$, triangles $-\mathrm{Re}=4000$; non-filled symbols $-T_{s} / T_{\infty}=1$, filled symbols $T_{s} / T_{\infty}=10$.
Figure 6. Pressure coefficient $C_{p}$ vs $\theta(\mathrm{rad}):$ (a) - $\mathrm{Ma}=5$, (b) $\mathrm{Ma}=10$, (c) - $\mathrm{Ma}=20$; circles $-\mathrm{Re}=0.1$, squares $-\mathrm{Re}=10$, triangles - $\mathrm{Re}=4000$; non-filled symbols $-T_{s} / T_{\infty}=1$, filled symbols $T_{s} / T_{\infty}=10$. 

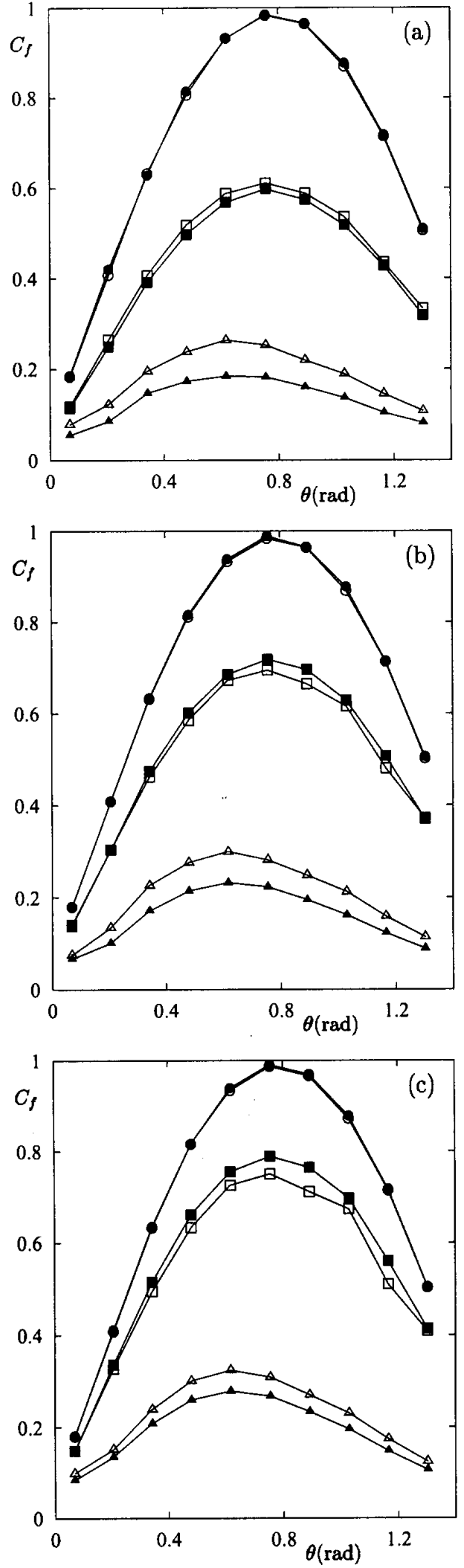
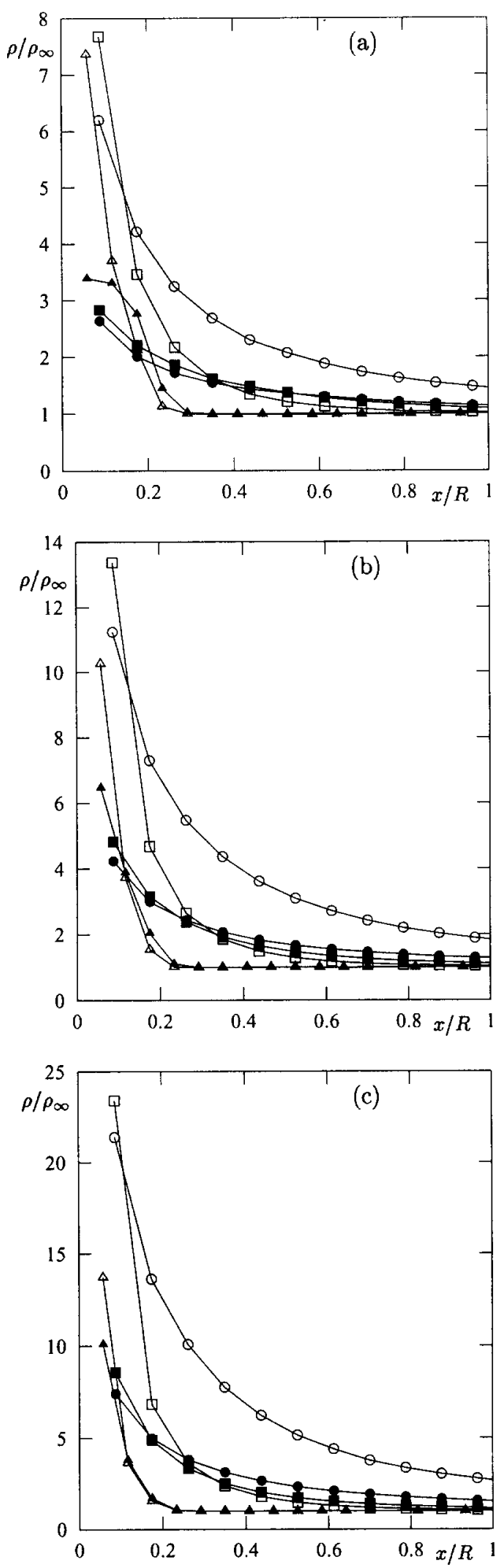

Figure 8. Density distribution in front of the satellite: (a) - Ma=5, (b) $-\mathrm{Ma}=10$, (c) - $\mathrm{Ma}=20$; circles $-\mathrm{Re}=0.1$, squares $-\mathrm{Re}=10$, triangles - Re=4000; non-filled symbols $-T_{s} / T_{\infty}=1$, filled symbols $T_{s} / T_{\infty}=10$

Figure 7. Friction coefficient $C_{f}$ vs $\theta(\mathrm{rad})$ : (a) - $\mathrm{Ma}=5$, (b) $\mathrm{Ma}=10$, (c) - $\mathrm{Ma}=20$; circles $-\mathrm{Re}=0.1$, squares $-\mathrm{Re}=10$, triangles - $\mathrm{Re}=4000$; non-filled symbols $-T_{s} / T_{\infty}=1$, filled symbols $T_{s} / T_{\infty}=10$. 

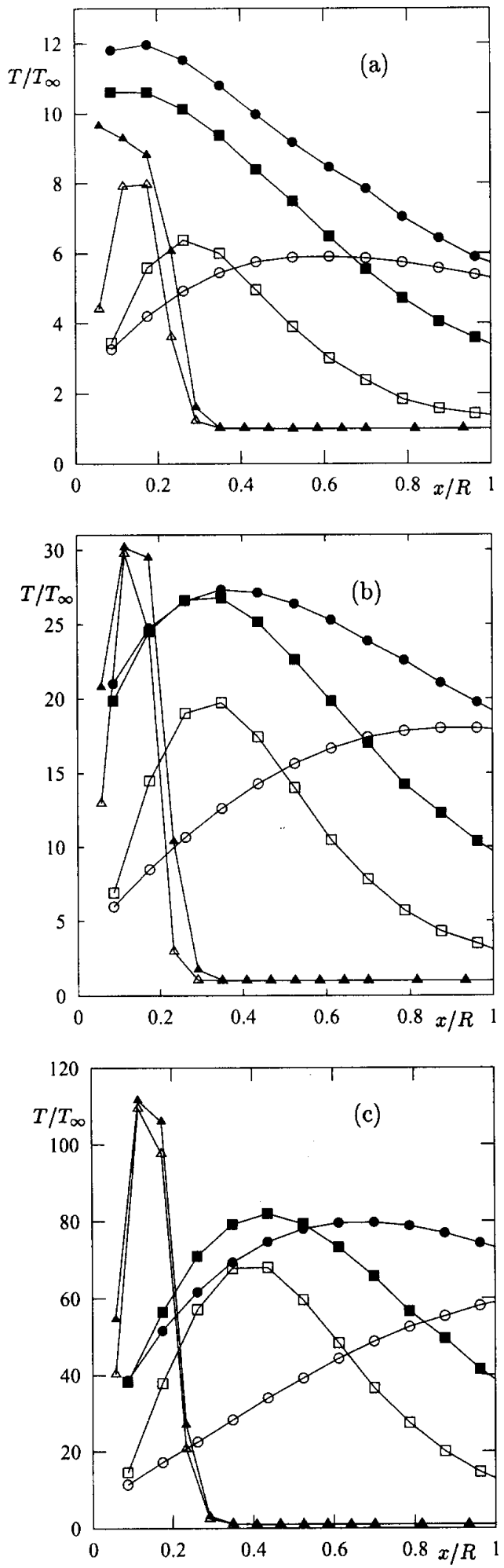

Figure 9. Temperature distribution in front of the satellite: (a) - $M a=5$, (b) - $M a=10$, (c) - $M a=20$; circles - $R e=0.1$, squares $\mathrm{Re}=10$, triangles $-\mathrm{Re}=4000$; non-filled symbols $-T_{s} / T_{\infty}=1$, filled symbols $-T_{s} / T_{\infty}=10$.
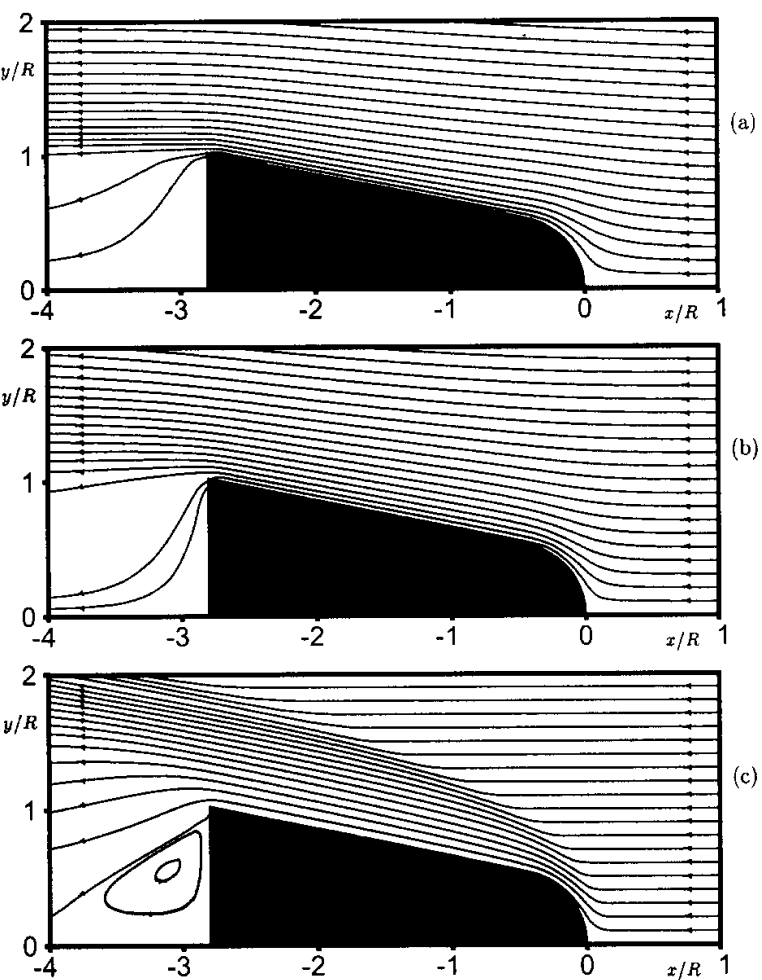

Figure 10. Streamlines at $\mathrm{Ma}=20$ : (a) $-\mathrm{Re}=0.1$, (b) $-\mathrm{Re}=10$, (c) $\mathrm{Re}=4000$.

The temperature distribution along the $x$ axis is presented in Fig. 9. Here, the shock wave is clearly observed in the hydrodynamic regime $(\operatorname{Re}=4000)$, i.e. the temperature of the gas drastically changed in the range $0<x / R<0.3$ reaching its maximum value in the point $x / R \approx 0.1$. In the free molecular regime $(\operatorname{Re}=0.1)$ the variation of the gas temperature is very slow. The influence of the satellite temperature upon the gas temperature is very weak in the hydrodynamic regime $(\mathrm{Re}=4000)$ and for the high values of the Mach number ( $\mathrm{Ma}=10$ and 20), while in all other situations this influence is strong.

The streamlines are given in Fig. 10 for $\mathrm{Ma}=20$, from which it can be seen the significant difference of the flowfield in the free molecular regime $(\operatorname{Re}=0.1)$ and in the hydrodynamic regime $(\mathrm{Re}=4000)$. First, at $\mathrm{Re}=4000$ the shock wave clearly divides the undisturbed gas and the disturbed one. Second, the vortices appear behind of the satellite in the hydrodynamic regime.

\section{Conclusions}

The aerothermodynamic characteristics necessary to realize the Brazilian project of reusable satellite SARA have been calculated for three values of the Mach number: 5, 10 , and 20 in the range of the Reynolds number from 0.1 to 4000 . This variation of the Reynolds number corresponds to the variation of the flow from the free molecular regime to the hydrodynamic one. The influence of the satellite temperature on aerothermodynamic characteristic was also investigated. For the high values of the Mach number (10 and 20) this influence is very weak and can be neglected, while at $\mathrm{Ma}=5$ some characteristics are affected by 
the satellite temperature. The most sensitive characteristic is the heat transfer coefficient $C_{H}$. More details on the aerothermodynamic characteristics can be found on the Web site http://fisica.ufpr.br/sharipov/ in the section "Scientific projects".

In the future the present work will be developed considering diatomic gases and taking into account the processes of dissociation, recombination, and ablation. These phenomena must be taken into account because of the high temperature of the gas in the shock wave appearing in front of the satellite.

\section{Acknowledgements}

This work is supported by Agência Espacial Brasileira (AEB, Brazil). The author also thanks the Conselho Nacional de Desenvolvimento Científico e Tecnológico (CNPq, Brazil) for the support of his work.

\section{References}

[1] M. N. Kogan, "Kinetic theory in aerothermodynamics," Prog. Aerospace Sci. 29, 271-354 (1992).

[2] V. M. Kotov, E. N. Lynchkin, and A. G. Reshetin, "An approximate method of aerodynamics calculation of complex shape bodies in a transition region," in Rarefied Gas Dynamics, (eds. O. M. Belotserkovskii, M. N. Kogan, S. S. Kutateladze, and A. K. Rebrov) (13th Int. Symp., USSR, 1982, Novosibirsk, 1985), Vol. 1, pp. 487-493.

[3] G. Koppenwallner, "Low Density Aerodynamics of Sattelites," in Rarefied Gas Dynamics, (eds. O. M. Belotserkovskii, M. N. Kogan, S. S. Kutateladze, and A. K. Rebrov) (13th Int. Symp., USSR, 1982, Novosibirsk, 1985), Vol. 1, pp. 385-399.

[4] A. B. Carlson and H. A. Hassan, "Direct simulation of reentry flows with ionization,” Tech. Rep., NASA (1990).

[5] V. K. Dogra, R. G. Wilmoth, and J. N. Moss, "Aerothermodynamics of a 1.6-meter-diameter sphere in hypersonic rarefied flow," AIAA Journal 30, 1789-1794 (1992).

[6] J. L. Potter and S. W. Peterson, "Local bridging to predict aerodynamic coefficients in hypersonic, rarefied flow," J. Spacecraft Rocket 29, 344-351 (1992).
[7] J. Allégre, A. Chpoun, M. Raffin, and J. C. Lengrand, "Experimental and numerical investigation of rarefied hypersonic flow around the ARD reentry module," in Rarefied Gas Dynamics, (ed. C. Shen), 20th Int. Symp., 1996 (Pekin University Press, Beijing (China), 1997), pp. 473-478.

[8] G. N. Markelov, A. N. Kudryavtsev, and M. S. Ivanov, "Numerical modeling of near-continuum hypersonic flow about a hollow cylinder flare," in Rarefied Gas Dynamics, (eds. R. Brun, R. Campargue, R. Gatignol, and J. C. Lengrand), 21st Int. Symp., France, 1998 (CEPAD, Toulouse, 1999), Vol. 2, pp. 647-654.

[9] P. A. Gnoffo, "Planetary-entry gas dynamics," Annu. Rev. Fluid Mech. 31, 459-494 (1999).

[10] G. J. LeBeau and F. E. Lumpkin III, "Application highlights of the DSMC analysis code (DAC) software for simulating rarefied gas flows," Comput. Methods Appl. Mech. Engrg. 191, 595-609 (2001).

[11] F. Sharipov, "Direct simulation Monte Carlo method applied to aerothermodynamics," J. Braz. Soc. Mechanical Sciences 23, 441-452 (2001).

[12] M. N. Kogan, Rarefied Gas Dynamics (Plenum, New York, 1969).

[13] C. Cercignani, The Boltzmann Equation and its Application (Springer, New York, 1988).

[14] C. Cercignani, Theory and Application of the Boltzmann Equation (Scottish Academic Press, Edinburgh, 1975).

[15] J. H. Ferziger and H. G. Kaper, Mathematical Theory of Transport Processes in Gases (North-Holland Publishing Company, Amsterdam, 1972).

[16] F. Sharipov and V. Seleznev, "Data on internal rarefied gas flows," J. Phys. Chem. Ref. Data 27, 657-706 (1998).

[17] G. A. Bird, Molecular Gas Dynamics and the Direct Simulation of Gas Flows (Oxford University Press, Oxford, 1996).

[18] O. V. Sazhin, S. F. Borisov, and F. Sharipov, "Accommodation coefficient of tangential momentum on atomically clean and contaminated surfaces," J. Vac. Sci. Technol. A 19, 24992503 (2001), erratum: 20 (3), 957 (2002). 